\title{
openheart Impact of transcatheter aortic valve implantation on the risk of mortality in patients with severe aortic valve diseases: a health insurance- based analysis
}

\author{
Alexander Barth, ${ }^{1,2}$ Seyrani Yücel, ${ }^{3}$ Hüseyin Ince, ${ }^{3}$ Gabriele Doblhammer ${ }^{1,2}$
}

\begin{abstract}
- Additional material is published online only. To view please visit the journal online (http://dx.doi.org/10.1136/ openhrt-2017-000756).
\end{abstract}

To cite: Barth A, Yücel S, Ince $H$ et al. Impact of transcatheter aortic valve implantation on the risk of mortality in patients with severe aortic valve diseases: a health insurance-based analysis. Open Heart 2018;5:e000756. doi:10.1136/ openhrt-2017-000756

Received 1 December 2017 Revised 7 March 2018 Accepted 21 March 2018
Check for updates

${ }^{1}$ Institute of Sociology and Demography, University of Rostock, Rostock, Germany ${ }^{2}$ Rostock Center for the Study of Demographic Change, University of Rostock, Rostock, Germany ${ }^{3}$ Divison of Cardiology, University Hospital Rostock, Rostock Germany

\section{Correspondence to} Alexander Barth; alexander. barth2@uni-rostock.de

\section{ABSTRACT}

Background Transcatheter aortic valve implantation (TAVI) is a minimally invasive treatment for aortic valve patients who are inoperable or have a prohibitively high surgical risk for surgical aortic valve replacement (SAVR). Most studies compare the efficacy of TAVI and SAVR, yet the assessment of TAVI for this group of patients requires more study.

Methods This quasiexperimental study compares TAVI cases (ages of 75-90 years, $n=187$ ) ex-post with a control group without implantation $(n=728,4: 1$ ratio intended). The control group was drawn randomly on the condition that it matches the TAVI cases based on age at aortic valve disease incidence, gender and comorbidity index. The mortality risk is analysed from incident diagnosis. Data were taken from three random samples of health claims data in Germany's largest public health insurance (Allgemeine Ortskrankenkassen) and cover the years 2004-2013 $(n=750000)$.

Results Compared with the medically treated control group with $6+$ comorbidities, medically treated patients with fewer comorbidities have half the mortality risk (HR $0.48,95 \% \mathrm{Cl} 0.34$ to $0.69, \mathrm{p}<0.001$ ). TAVI patients with fewer than six comorbidities show a mortality risk half that (HR $0.23,95 \% \mathrm{Cl} 0.09$ to $0.63, \mathrm{p}=0.004$ ). TAVI patients with $6+$ comorbidities do not benefit from TAVI compared with the control group with $6+$ comorbidities (HR $0.99,95 \% \mathrm{Cl} 0.71$ to $1.36, p=0.93$ ).

Conclusion TAVI is an effective therapy for aortic valve disease patients with few comorbidities; it is not effective for patients with a high comorbidity burden. Careful assessment of the individual patient in terms of comorbidities is important for a beneficial outcome.

\section{BACKGROUND}

Symptomatic aortic valve diseases are common in older people and are associated with a high mortality risk. Treatment of patients with severe symptomatic aortic stenosis has rapidly changed in the past decade. Transcatheter aortic valve implantation (TAVI) has become an established therapeutic option for inoperable and high surgical risk patients. Various studies
Key messages

What is already known about this subject? For inoperable or high surgical risk patients with aortic valve stenosis, TAVI is generally considered to be more beneficial than standard medical treatment. However, additional information on the role of comorbidities in relation to the benefits of transcatheter aortic valve implantation (TAVI) would be helpful.

What does this study add?

Using a quasiexperimental retrospective design based on routine observational health claims data, it provides evidence that TAVI is beneficial compared with standard treatment, but only for patients without too many comorbidities.

How might this impact on clinical practice? Patient selection is key to ensure benefits for TAVI patients compared with standard treatment; it should include the type and the number of comorbidities.

compare survival outcomes between TAVI and surgical aortic valve replacement (SAVR) patients. ${ }^{1-9}$ These studies provide concurrent evidence that TAVI is at least not inferior to SAVR and is often more favourable in terms of all-cause and cardiovascular mortality in short and longer term follow-ups. TAVI was also not found to be inferior to SAVR in terms of valve function, health-related quality of life increase, reduction of symptoms caused by aortic valve disease or in terms of complications related to aortic valve disease such as stroke or myocardial infarction. More evidence on the outcomes of TAVI compared with medical treatment in similar patients would be helpful, because there is currently not much literature on whether and to what degree previously inoperable patients can profit from TAVI 


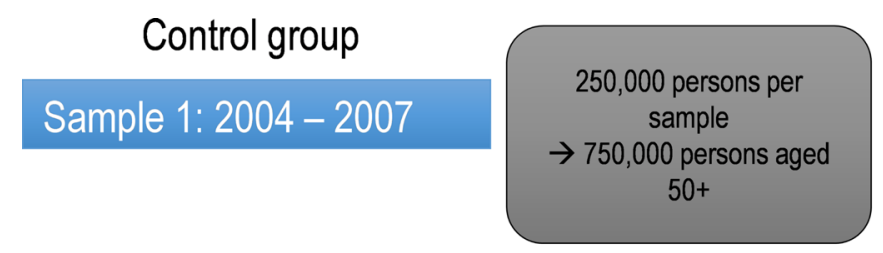

TAVI
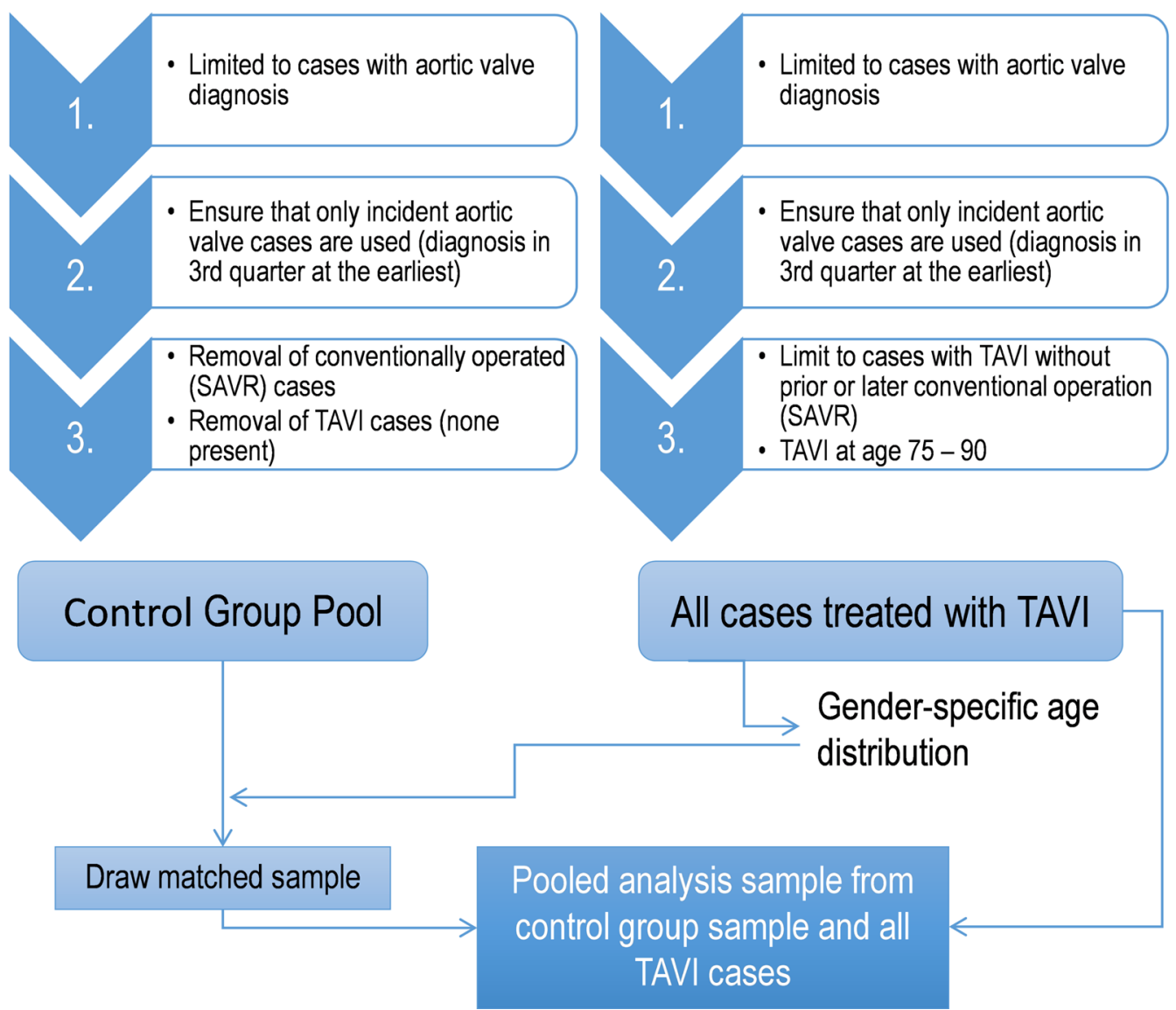

Figure 1 Analysis sample generation. SAVR, surgical aortic valve replacement; TAVI, transcatheter aortic valve implantation.

\section{HYPOTHESES}

Compared with non-operative treatment, TAVI is said to be beneficial for patients with aortic valve diseases in terms of reduced mortality risk. TAVI is expected to yield better results for patients who generally are in better health compared with patients with several comorbidities. Patient selection was an important factor for positive outcomes in most previous studies, thus the benefit of TAVI compared with non-operative treatment is expected to depend strongly on the patients' medical characteristics in terms of comorbidities.

\section{DATA}

This study is based on longitudinal routine data from Germany's largest public health insurance, Allgemeine Ortskrankenkassen (AOK). A total of three random samples, each consisting of 250000 persons aged $50+$ years, were drawn. Each sample spans 4 years, starting at three different times: sample 1 starts at the beginning of 2004 and ends in 2007, sample 2 covers 2007-2010 and sample 3 comprises 2010-2013. Cases were eligible to be drawn regardless of whether medical treatment was actually received. The data include information on sex, dates of birth and death and a complete record of the inpatient and outpatient diagnoses and operative procedures, which are covered by health insurance and are available on a quarterly basis. Data do not include exact clinical measurements and indicators such as aortic valve diameter or clinical risk calculators such as the EuroScore or STS-Score. The samples were drawn from all insurants aged 50 years or older, without special concern for any specific diseases or procedures such as aortic valve diseases or TAVI.

\section{VARIABLES AND DEFINITIONS}

In order to properly select TAVI and control cases, those aortic valve diseases that are relevant for TAVI treatment must be identified. Diagnoses are available as ICD-10-GM codes. We identified acquired rheumatic and non-rheumatic aortic valve diseases including stenosis and insufficiency (I06 and I35) and aortic valve stenosis and insufficiency from birth (Q230 and Q231) as being relevant for 
TAVI. To ensure the validity of diagnoses, only confirmed outpatient diagnoses made by internal medicine specialists (primarily cardiologists) and confirmed stationary discharge diagnoses are used.

The health status of aortic valve patients is differentiated based on an additive comorbidity index, which includes 22 primarily chronic diseases relevant at older ages. Included diseases are atherosclerosis, breast cancer, colorectal cancer, lung cancer, pancreatic cancer, prostate cancer, stomach cancer, cerebrovascular diseases, dementia, depression, diabetes, lower extremity injuries, other external injuries, hypertension, ischaemic diseases, kidney diseases, alcoholic liver diseases, lung diseases, myocardial infarction, nervous diseases, Parkinson's disease and vascular diseases. They are measured by using confirmed diagnoses from outpatient physicians and inpatient discharge diagnoses. A disease is considered to be present from the first diagnosis onwards. For the purpose of drawing the control group sample, the comorbidity index was partitioned into quintiles. For the statistical analysis, it was further condensed into two groups: up to five and six or more diseases. Age is used as single year age when drawing the control group and is included in the statistical analysis in 5-year age-groups (75-79, $80-84,85-89$ and $90+$ years). Sex (male or female) is a binary variable. Death is a binary variable indicating the time of death and is used as the primary outcome.

\section{STUDY DESIGN AND SAMPLE SELECTION}

The study is retrospective, combining the TAVI cases ex-post with a control group without implant treatment. The control group was randomly drawn on the condition that its structure matched the TAVI cases based on age at aortic valve disease incidence, gender and comorbidity index. A common disadvantage of quasiexperimental designs is that the subjects are able to admit themselves into treatment or non-treatment groups. This does not apply in our case, as the control group is drawn from sample 1. The TAVI (treatment) group, in contrast, are only found from 2008 onwards, in samples 2 (2007-2010) and 3 (2010-2013); sample 1 (2004-2007) contains no TAVI cases.

Several steps are necessary to define the analysis sample (summarised in figure 1). To select eligible TAVI cases, samples 2 (2007-2010) and 3 (2010-2013) are reduced to cases with at least one diagnosis of diseases relevant for aortic valve replacement. To compare the development of TAVI and control cases from the first diagnosis onwards, only those cases with incident aortic valve diseases are considered. Thus, only cases with at least two quarters without any aortic valve disease diagnosis at the beginning of the observation period are used, while cases with a diagnosis within the first two quarters are rejected. Cases with an incident diagnosis in the last available quarter are rejected as well in order to ensure the possibility of at least one quarter of follow-up. Finally, only those cases that received TAVI between the ages of 75 and 90 years are considered. Supplementary appendix table A-1 shows the composition of the TAVI group by sex, age and quintiles of the comorbidity index at the time of incident aortic valve disease diagnosis. The TAVI group consists of 187 cases, two-thirds of whom are female. Most cases are found in the ages between 79 and 87 years, and men are slightly younger than women.

Control cases are drawn randomly from the control group pool. This pool contains all cases with incident diagnosis in sample 1 (2004-2007) without SAVR or TAVI, except for those with a diagnosis in the first two quarters to ensure incident diagnosis, similar to the TAVI group. To match the control cases to the TAVI group, they are drawn under the condition that the control sample matches the composition of the TAVI group in terms of sex, age and comorbidity index at the time of incident aortic valve diagnosis. This was accomplished by randomly selecting the required number of non-implant cases separately for each combination of sex, age at incident diagnosis and comorbidity index score, resulting in a total of 160 subsamples (2 genders, 16 ages and 5 comorbidity index scores), which were combined to form the control group. This makes it possible to study survival outcomes from the point of incidence onwards, because the starting point for the TAVI and the control group is identical. Four control cases are drawn for each TAVI case. Supplementary appendix table A-2 shows the composition of the control group by sex, age at incidence and comorbidity index score, indicating that nearly all subsamples could be completely filled. The control group covers $97.3 \%$ of the intended composition. Thus, a very high quality control sample was drawn and pooled with all TAVI cases, resulting in a sample with a total of 915 persons. Supplementary appendix table A-3 shows the prevalence of individual comorbidities in the TAVI and control groups at the time of incident aortic valve disease diagnosis. For 6 of 22 comorbidities, a significant difference $(p<0.05)$ of prevalence was found between the TAVI and control groups. Three comorbidities were found significantly more often in the control group (lower extremity injuries, cerebrovascular and nervous diseases), and three more frequently in the TAVI group (diabetes, ischaemic heart diseases and atherosclerosis). An overview of the process leading to the final analysis sample is shown in figure 1.

\section{STATISTICAL ANALYSIS}

Based on the pooled analysis sample, descriptive Kaplan-Meier survival analysis and multivariate Cox proportional hazard survival regression analyses are performed. Multivariate models are adjusted for TAVI and control group cases with either up to five or six or more comorbidities and controlled for age and sex. Process time is defined as years since incidence of aortic valve diagnosis. Thus, patients enter at different ages but always at the time of incident aortic valve disease diagnosis. Sex is time invariate; all other covariates 
Table 1 Sample overview for TAVI and control group cases by comorbidity index

\begin{tabular}{lccc}
\hline & $\begin{array}{l}\text { Person- } \\
\text { years }\end{array}$ & Deaths & $\begin{array}{l}\text { Rate per 1000 } \\
\text { person-years }\end{array}$ \\
\hline Control (all) & 1077.6 & 191 & 177.2 \\
\hline TAVI (all) & 311.5 & 52 & 166.9 \\
$\begin{array}{l}\text { Control (0-5 } \\
\text { comorbidities) }\end{array}$ & 339.8 & 38 & 111.8 \\
$\begin{array}{l}\text { Control (six or more } \\
\text { comorbidities) }\end{array}$ & 737.8 & 153 & 207.4 \\
$\begin{array}{l}\text { TAVI (0-5 comorbidities) } \\
\text { TAVI (six or more }\end{array}$ & 75.6 & 4 & 52.9 \\
comorbidities) & 235.9 & 48 & 203.5 \\
Total & 1389.1 & 243 & 174.9 \\
\hline
\end{tabular}

TAVI, transcatheter aortic valve implantation.

are time variate. All cases are included until censoring (end of observation period or leaving the AOK insurance) or death, whichever occurred first.

\section{RESULTS}

Table 1 shows a descriptive overview of mortality after incident aortic valve diagnosis in the analysis sample for the TAVI and control groups in total and by comorbidity index.

In total, 1389 person-years are available in the pooled analysis sample, during which 243 deaths occurred, resulting in a mortality rate of 174.9 per 1000 personyears. Due to the intentionally large control group, its time at risk is larger than for the TAVI cases (about 1080 and 310 person-years, respectively). The control group has 191 deaths and shows a mortality rate of 177.2 per 1000 person-years. The entire TAVI group has 52 deaths and a mortality rate of 166.9 per 1000 person-years. Subdividing both groups by the number of comorbidities reveals that they affect mortality for both groups markedly. The control group shows mortality rates of 111.8 (38 deaths) for patients with up to five comorbidities and 207.4 (153 deaths) for patients with six or more comorbidities, while the TAVI group shows mortality rates of 52.9 (4 deaths) and 203.5 (48 deaths) per 1000 person-years, depending on the number of comorbidities. Thus, in a descriptive analysis, the number of comorbidities is a very important factor for the mortality of TAVI and control group patients. TAVI patients with few comorbidities show the lowest mortality rate, while patients with more comorbidities do not show markedly different mortality rates. This suggests that, for patients who do not suffer from many comorbidities, TAVI could lead to better outcomes compared with conventional treatment.

Table 2 depicts the mortality rates for the two remaining covariates that are included in the multivariate models: sex and age. Results are presented separately for the TAVI and control groups. For both, women show a lower mortality rate than men, with the highest mortality rate for men with TAVI. For the non-operatively treated control group, higher age is associated with higher mortality. For TAVI patients, this relationship is inverse: older TAVI patients show lower mortality than younger TAVI patients, with the exception of the highest age group. This might be related to stricter patient selection for TAVI at higher ages, so that only comparatively healthy, less multimorbid older patients with a positive prognosis receive TAVI.

Figure 2 shows the Kaplan-Meier survival curves for the TAVI and the control groups by number of comorbidities, the number of cases at risk over time and the result of the generalised Wilcoxon test for equality of survivor functions. Analogous to the descriptive overview, TAVI shows a higher share of survivors over the entire observation period for patients with up to five comorbidities, while there is no apparent benefit for the patients with more comorbidities. The Wilcoxon test indicates that the survivor functions are not equal.

HRs obtained from a multivariate Cox proportional hazard model with death as outcome are shown in table 3 . The model includes TAVI or control group membership with up to five or six or more comorbidities, sex and 5 -year age groups (the highest group is open ended).

Table 2 Sample overview of covariates for control and TAVI groups

\begin{tabular}{|c|c|c|c|c|c|c|}
\hline & \multicolumn{3}{|l|}{ Control } & \multicolumn{3}{|l|}{ TAVI } \\
\hline & Person-years & Deaths & $\begin{array}{l}\text { Rate per } 1.000 \\
\text { person-years }\end{array}$ & Person-years & Deaths & $\begin{array}{l}\text { Rate per } 1.000 \\
\text { person-years }\end{array}$ \\
\hline Male & 355.2 & 68 & 191.4 & 94.0 & 22 & 234.0 \\
\hline Female & 722.4 & 123 & 170.3 & 217.5 & 30 & 137.9 \\
\hline $75-79$ & 222.6 & 26 & 116.8 & 53.6 & 15 & 279.7 \\
\hline 80-84 & 470.8 & 80 & 169.9 & 133.6 & 21 & 157.2 \\
\hline $85-89$ & 339.5 & 71 & 209.1 & 103.0 & 13 & 126.2 \\
\hline $90+$ & 44.6 & 14 & 313.7 & 21.3 & 3 & 140.9 \\
\hline Total & 1077.6 & 191 & 177.2 & 311.5 & 52 & 166.9 \\
\hline
\end{tabular}

$\mathrm{TAVI}$, transcatheter aortic valve implantation. 


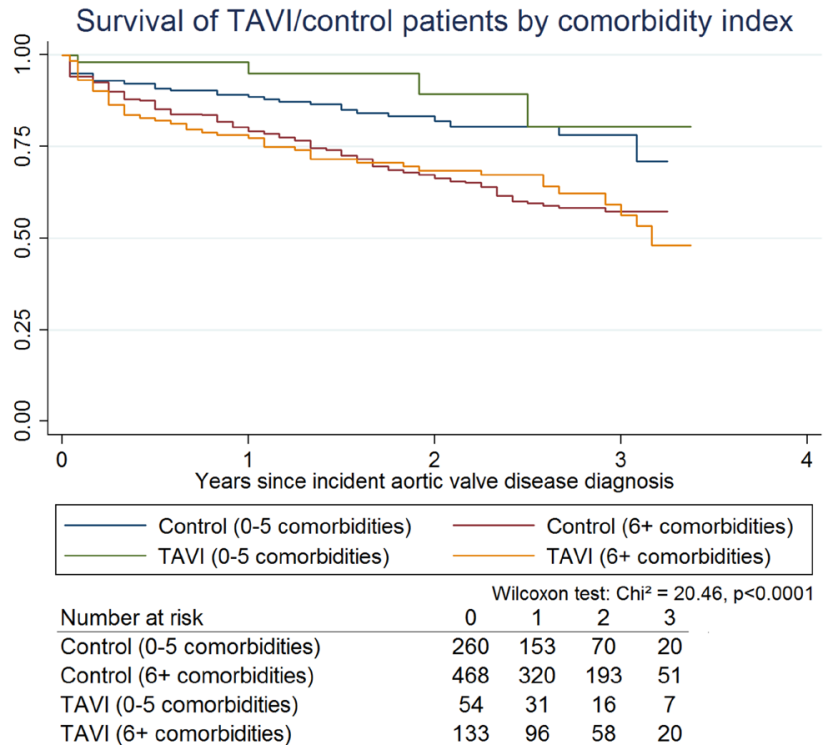

Figure 2 Kaplan-Meier survival curves for the TAVI and control group by number of comorbidities. TAVI, transcatheter aortic valve implantation.

As in the descriptive results, the categorised number of comorbidities markedly affects the mortality risk of aortic valve patients with and without TAVI. Controlled for sex and age and in comparison with the non-operatively treated control group containing patients with six or more comorbidities, the control group cases with five or fewer comorbidities show about half the mortality risk (HR $0.48,95 \%$ CI 0.34 to $0.69, \mathrm{p}<0.001$ ). TAVI patients with five or fewer comorbidities show a mortality risk about half as large as the respective control group (HR $0.23,95 \%$ CI 0.09 to $0.63, p=0.004)$. However, TAVI patients with six or more comorbidities do not show a different mortality risk than the control group patients with the same comorbidity profile (HR $0.99,95 \%$ CI 0.71 to $1.36, \mathrm{p}=0.93$ ). This indicates that TAVI is an effective

Table 3 HRs of death by TAVI/control group, sex, age and comorbidities

HR P values $95 \% \mathrm{CI}$

Control group/TAVI by number of comorbidities (reference: control group, 6 or more comorbidities)

Control group (up to five

$\begin{array}{llll}\text { comorbidities) } \quad 0.48 & <0.001 & 0.34 \text { to } 0.69\end{array}$

$\begin{array}{llll}\text { TAVI (up to five } & & & \\ \text { comorbidities) } & 0.23 & 0.004 & 0.09 \text { to } 0.63\end{array}$

TAVI (six or more

$\begin{array}{llll}\text { comorbidities) } & 0.99 & 0.93 & 0.71 \text { to } 1.36\end{array}$

$\begin{array}{llll}\text { Female (ref: male) } & 0.72 & 0.020 & 0.55 \text { to } 0.95\end{array}$

Age (ref: $75-79$ years)

$\begin{array}{llll}80-84 & 1.24 & 0.242 & 0.86 \text { to } 1.79 \\ 85-89 & 1.74 & 0.005 & 1.18 \text { to } 2.56 \\ 90+ & 2.67 & 0.001 & 1.48 \text { to } 4.81\end{array}$

$\mathrm{n}=915$, LR (likelihood ratio test) $=42.2 ; \mathrm{p}<0.001$.

$\mathrm{TAVI}$, transcatheter aortic valve implantation. therapy for patients with symptomatic aortic valve disease and few comorbidities, and it is not effective for patients with a high comorbidity burden. As seen in the descriptive results, females show a significantly lower mortality risk than men (HR 0.72, $95 \%$ CI 0.55 to $0.95, \mathrm{p}=0.02$ ). Higher HRs for higher age groups indicate increasing mortality risks at higher ages: HR 1.74 for ages $85-89$ years $(95 \%$ CI 1.18 to $2.56, \mathrm{p}=0.005$ ) and HR 2.67 (95\% CI 1.48 to 4.81 , $\mathrm{p}=0.001$ ) for ages 90 years and higher. Only the effect for the second highest age group (80-84) is insignificant (HR $1.24,95 \%$ CI 0.86 to $1.79, \mathrm{p}=0.24$ ). An interaction between the control/TAVI by comorbidity variable and sex (not shown) revealed no significant effects, thus the lower mortality risk for females is present independent of TAVI or conventional treatment and comorbidity profile. To assess the role of specific comorbidities, we also calculated an additional model for each comorbidity included in the comorbidity index. While their results indicated that for none of the comorbidities a significant difference $(p<0.05)$ between TAVI and control patients with each individual comorbidity existed, for most comorbidities TAVI at least tended to insignificantly reduce mortality risk. Myocardial infarction, alcoholic liver disease, nervous diseases, external injuries, colorectal cancer and lung diseases were exceptions, because TAVI tended to show no benefits compared with medical treatment. Thus, generally, the benefit of TAVI for patients with less than six comorbidities shown in table 3 is not related to one or more specific comorbidities but to the burden of multiple comorbidities.

\section{DISCUSSION}

The most prominent study comparing TAVI to medical treatment with long-term data is the 5-year outcome analysis of the Placement of AoRTic TraNscathetER Valve Trial (PARTNER) $1 \mathrm{~B}$ trial. ${ }^{10}$ It concludes that TAVI performs favourably in terms of mortality and symptom reduction after 5 years compared with medical treatment and suggests that TAVI should always be considered for patients who are unable to undergo SAVR; however, patient selection is key to ensure beneficial outcomes.

The only other study of TAVI compared with non-operative treatment not based on PARTNER trial data also indicates that TAVI is able to reduce mortality. ${ }^{11}$ However, the control group in this study includes only people who were ineligible for TAVI, which is a potential selection effect not present in our study. In this study, compared with the control group that received neither TAVI nor SAVR, TAVI shows a significantly reduced mortality risk for patients who do not suffer from too many comorbidities $(<6$ comorbidities). Patients who received TAVI but suffer from a high number of comorbidities do not significantly differ from the control group in terms of mortality. Based on our results, the successful outcome of TAVI in terms of reduced mortality is primarily dependent on the number of the patient's comorbidities. 


\section{STRENGTHS AND WEAKNESSES}

Clinical studies can rely on clinical measures of overall operative risk such as Society of Thoracic Surgeons Predicted Risk of Mortality (STS) scores and on parameters which characterise the severity of aortic valve stenosis such as valve orifice area or pressure gradients. This information would be very useful to match treatment and control patients, regardless of whether the control group consists of conventionally implanted SAVR or non-operatively treated patients. Our data only contain information on medical procedures that are covered by the health insurance. For this reason, the selection of the control group had to be performed based on sex, age and number of comorbidities at the time of incident aortic valve disease diagnosis. Because the data do not contain disease-specific causes of death, it is unclear if patients died because of aortic valve insufficiency or due to other causes. Thus, we use all-cause mortality as endpoint.

The samples of health claims data used here are large, include patients from all parts of Germany and include very detailed information on diagnoses and procedures from both the ambulatory and stationary sectors. The quasiexperimental design relying in part on data from a point in time where TAVI was not yet available does not cause any ethical problems, as no available treatment was withheld from the control group patients. Self-selection of possible control group patients into either TAVI or conservative treatment options was not possible for the same reason, because the choice to undergo TAVI was non-existent at the time and SAVR was generally performed whenever possible, leaving patients unfit for SAVR as control group candidates, which is exactly the group now eligible for less invasive TAVI. Unlike clinical studies that are generally limited to a few participating centres and patients specifically selected for the study, our data reflect the real-world usage of TAVI all across Germany.

\section{CONCLUSION}

Minimally invasive TAVI has proven to be an effective therapy in older and often multimorbid high-risk patients, who cannot undergo SAVR. Data from RCT and clinical studies even show lower mortality rates in TAVI in some subgroups. ${ }^{210} 12$ However, the potential benefits of TAVI for the previously inoperable elderly frail population are less well studied.

To increase the benefits of TAVI for inoperable and high-risk patients, the assessment of the individual case in terms of comorbidities is the most important factor, because a higher number of comorbidities decreases the benefit of TAVI. The patient selection seems the key to ensuring beneficial outcomes.

In our study, we found that TAVI showed a significant reduction in mortality risk in patients with fewer comorbidities compared with the untreated control group with fewer comorbidities. In patients with increased burden of comorbidities, TAVI shows no significant reduction in mortality risk, indicating that patient selection is a key factor for successful TAVI outcome. Based on our results, which rely on comorbidities instead of clinical parameters of operative risk or aortic valve disease severity, TAVI should be considered for patients with fewer than six severe comorbidities. Especially patients with myocardial infarction, alcoholic liver disease, nervous diseases, external injuries, colorectal cancer or lung diseases should be assessed carefully.

Future research focusing on the role of TAVI benefits in the presence of specific comorbidities might help to improve the assessment process of potential TAVI patients.

Contributors $A B$ carried out the statistical analysis and drafted the manuscript; $A B$ and $S Y$ revised the manuscript. $A B$ prepared the data. All authors conceived the study; $A B$ implemented the study. All authors read and approved the final manuscript.

Funding This study was funded by Bundesministerium für Bildung und Forschung. Competing interests None declared.

Patient consent Not required.

Provenance and peer review Not commissioned; externally peer reviewed.

Open Access This is an Open Access article distributed in accordance with the Creative Commons Attribution Non Commercial (CC BY-NC 4.0) license, which permits others to distribute, remix, adapt, build upon this work non-commercially, and license their derivative works on different terms, provided the original work is properly cited and the use is non-commercial. See: http://creativecommons.org/ licenses/by-nc/4.0/

(c) Article author(s) (or their employer(s) unless otherwise stated in the text of the article) 2018. All rights reserved. No commercial use is permitted unless otherwise expressly granted.

\section{REFERENCES}

1. Nagaraja V, Raval J, Eslick GD, et al. Transcatheter versus surgical aortic valve replacement: a systematic review and metaanalysis of randomised and non-randomised trials. Open Heart 2014;1:e000013-18.

2. Möllmann $\mathrm{H}$, Bestehorn $\mathrm{K}$, Bestehorn $\mathrm{M}$, et al. In-hospital outcome of transcatheter vs. surgical aortic valve replacement in patients with aortic valve stenosis: complete dataset of patients treated in 2013 in Germany. Clin Res Cardiol 2016;105:553-9.

3. Panchal HB, Ladia V, Desai S, et al. A meta-analysis of mortality and major adverse cardiovascular and cerebrovascular events following transcatheter aortic valve implantation versus surgical aortic valve replacement for severe aortic stenosis. Am J Cardiol 2013;112:850-60.

4. Cao C, Ang SC, Indraratna P, et al. Systematic review and metaanalysis of transcatheter aortic valve implantation versus surgical aortic valve replacement for severe aortic stenosis. Ann Cardiothorac Surg 2013;2:10-23.

5. Kodali SK, Williams MR, Smith CR, et al. Two-year outcomes after transcatheter or surgical aortic-valve replacement. $N$ Engl J Med 2012;366:1686-95.

6. Leon MB, Smith CR, Mack MJ, et al. Transcatheter or Surgical Aortic-Valve Replacement in Intermediate-Risk Patients. N Engl J Med 2016;374:1609-20.

7. Smith CR, Leon MB, Mack MJ, et al. Transcatheter versus surgical aortic-valve replacement in high-risk patients. $N$ Engl J Med 2011;364:2187-98.

8. Takagi H, Niwa M, Mizuno $\mathrm{Y}$, et al. A meta-analysis of transcatheter aortic valve implantation versus surgical aortic valve replacement. Ann Thorac Surg 2013;96:513-9.

9. Reynolds MR, Magnuson EA, Wang K, et al. Health-related quality of life after transcatheter or surgical aortic valve replacement in highrisk patients with severe aortic stenosis: results from the PARTNER (Placement of AoRTic TraNscathetER Valve) Trial (Cohort A). J Am Coll Cardiol 2012;60:548-58.

10. Kapadia SR, Leon MB, Makkar RR, et al. 5-year outcomes of transcatheter aortic valve replacement compared with standard 
treatment for patients with inoperable aortic stenosis (PARTNER 1): a randomised controlled trial. Lancet 2015;385:2485-91.

11. Rajani R, Buxton W, Haworth P, et al. Prognostic benefit of

transcatheter aortic valve implantation compared with medical therapy in patients with inoperable aortic stenosis. Catheter Cardiovasc Interv 2010;75:1121-6.

12. Barker CM, Reardon MJ. The CoreValve US pivotal trial. Semin Thorac Cardiovasc Surg 2014;26:179-86. 